\section{PNEUMOCOCCIC ARTHRITIS.}

BY EDWARD J. CATE, M.D., M.R.C.P. LoND., PHYSICIAN TO THE ROYAL UNITED HOSPITAL, BATH.

IT would seem that hitherto no observations have been made in this country of the occurrence of arthritis associated with the presence of the pneumococcus in the affected joint, and although the association is probably a very uncommon one, yet, having regard to the comparative rarity of bacteriological examination of morbid fluids, one cannot but suppose that such cases may have occurred and yet escaped detection. The case herein related led to an examination of the literature of the subject, and as some 30 instances are recorded by continental observers there exists sufficient material to form the basis of a fairly complete picture of the disease. The observations up to 1899 have been collected by Dr. L. Leronx ${ }^{1}$ of Paris and are embodied in an excellent monograph. These cases, together with three others recorded during the past year, are analysed in the accompanying table. Following $D$. Leroux no account has been taken of records prior to Weichselbaum's ${ }^{2}$ observations in 1888, although not a few instances are scattered through the periodical literature of the past century in which arthritis has supervened during or shortly after an attack of pneumonia ${ }^{3}$ and in some of these the clinical accounts are so definite and conform so closely to what we now know of the course of the affection as to leave little doubt of their pneumococcic nature. Noticeably is this the case in some records of Grisolle. ${ }^{4}$ Four patients, he says, who had never suffered from any form of articular or muscular rheumatism were attacked either early in the course of a pneumonia or shortly after its subsidence with acute arthritis, affecting in one case the two shoulders, in another the right knee, in the third the left ankle, and in the fourth the right wrist and the left knee. The last case was examined after death and both the affected joints were distended by odourless pus and the synovial fringes were injected. The joint affection was always a continuous one with none of the tugitive character of true rheumatism, and after discussing the relations with this latter disease Grisolle came to the conclusion that the nature of the two affections was quite distinct. He could not, however, exclude the possibility of a pyæmic process. In this country Wilson Fox, ${ }^{5}$ after referring to Grisolle's cases, mentions that of a man of dissipated habits who was under the care of Dr. Russell Reynolds for pnenmonia. On the day after the crisis there occurred a slight rise of temperature and an effusion into the knee-joint. 'The pneumonia resolved perfectly, but the arthritis became chronic. These cases occurred prior to the discovery of the pneumococcus and the true significance of the association could not therefore be appreciated. It was not until 1833 and following years that the infective nature of pneumonia, together with the causative agency of the pneumococcus in that disease, was worked out by Talamon, Stern berg, Fraenkel, Weichselbaum, and other observers, and shortly after the earliest publication of this association was discovered the pathogenic efficacy of the same organism in the production of inflammations of various serous membranes and of the joints. Attacks of arthritis associated with pneumococci were recorded in 1884 and 1885, but the first case to be satisfactorily established was that of Weichselbaum which has been already referred to and which was published in 1888. As in the case recorded in this paper the observation related to a suppurative artbritis of the shoulder.

In April, 1900, I was asked to see a man suffering from pneumonia. I found that the patient was a stout, burly farmer, 51 years of age, of alcoholic habits. When I saw him be was moribund. The account of his illness given to me was

1 Les Arthrites à Pneumocoques, Paris, J. Rousset, 1899. I have made the treest use of the observations collected in Leroux's work.

2 Teber seltene Lokalisationen des Pneumonischen Virus, Wiener Klinische Wochenschrift, 1888, Nos. 28-32.

3 No reference is made here to the well-known association of pneumonia or of physical signs indistinguishable from those of pneumonia at the left base with acute pericardial effusion in the course of rheumatic fever. In the recorded cases the general symptoms and characteristic sputa of pneumonia have generally been absent and the diagnosis made from the physical signs alone. Uf. Southey: THE LANCET, Dec. 9th, 1882, p. 9R3: Choadle : THE LAxclT, May 5tb, 1888, p. 861.

Traité de la Pneumonie, second edition, 1864 . p. 394 that he had been seized 11 days before with rigor, pain in his side, and other symptoms of pneumonia, with physical signs of consolidation confined to the lower lobe of the right lung. After a severe attack with much delirium crisis supervened on the sixth day of his disease and for 12 hours his temperature was normal or below and his general condition improved. In the evening of the same day his temperature again rose to $103.5^{\circ} \mathrm{F}$, violent delirium set in, and on the following day were detected signs of consolidation at the base of the left lung. It was on the fifth day of this recrudescence of the disease that I saw him, when he was in a state of coma-vigil, with a pulse of 160 or over, prof usely perspiring, with dilated pupils, and evidently near bis end. He was too ill to be examined, but on looking at the front of his thorax I was immediately struck with the condition of his left shoulder. This was swollen, deep red, and cdematous, and slight movement of the upper extremity evoked, even in his well-nigh unconscious state, signs of suffering and remonstrance. I was told that five days earlier, during violent delirium, and while struggling with his attendants, be complained that his arm had been twisted. The next day he complained persistently of pain in his shoulder, being delirious for most of the day. On the following day (the third of the injury and ninth of his disease) his shoulder was noticed to be red and swollen and the least movement led to violent exclamations. The condition had persisted, with the appearance of œdema, until the time of my visit. He died within an hour, and shortly after death, no complete post-mortem examination being possible, the skin over the deltoid muscle was seared with a hot iron and a sterilised needle was thrust into the joint. A syringeful of thick greenish creamy pus was drawn off. This contained an abundance of pneumococci, in pairs and short chains of three or four elements, distinctly encapsuled and in pare culture. The leucocytes were polynuclear. The cocci stained well by Gram's method, and when stained by Ziehl's solution and partly decolourised in 1 per cent. acetic acid the capsules were very well shown. Typical dew-drop cultures were obtained on agar and blood serum. Its virulence to mice or rabbits was not tested.

This is a very typical case of the acute suppurative form of pneumococcic arthritis and it illustrates many of the ordinary features of the disease; its occurrence as a complication or immediate sequel of pneumonia, its tendency to affect a joint previously damaged by trauma or disease, a "locus minoris resistentiæ," its acute course, "its early suppuration, and its commonly fatal event.

Of the 31 cases analysed in the table 23 patients died and eight recovered. The vast majority of the cases occurred in immediate association with pneumonia (28 out of 31 ), the symptoms of arthritis usually following the onset of the pneumonia at intervals varying from a few days to a fortnight. In two instances (observations of Boulloche and oliva) the arthritis is said to have preceded the pneumonia by an interval of three days in one case and seven days in the other. Both these cases occurred in children, being the only instances recorded under 30 years of age, and that of Oliva is so unlike most of the other cases in the table that it will be referred to later in connexion with a case of multiple arthritis which the writer has hesitated to include. Three cases are described as occurring independently of pneumonia. The first, related by Griffon ${ }^{6}$ in 1896 , was that of a woman, aged 71 years, who was admitted comatose with a temperature of $104^{\circ} \mathrm{F}$. and a suppurative arthritis of the right ankle. The joint was incised on the same night, but the patient succumbed on the following day. In addition to extensive suppuration in the ankle and calcaneo-astragaloid joints and the neighbouring synovial sheaths, the necropsy disclosed meningitis of the vertex and a recent vegetating endocarditis. Pneumococci were demonstrated in the pus of the affected joint, in the cardiac vegetation, and in the purulent exudation on the meninges. The second case is related by Widal and Meslay in 1896. The patient, an adult male who was the victim of chronic impregnation with lead, complained of dysphagia and arthritis of the first metatarso-phalangeal joint of the left foot; the joint was painful and slightly swollen, and the skin over it was hot and red. The temperature varied from $101^{\circ}$ to $102 \cdot 2^{\circ} \mathrm{F}$. At the end of a fortnight the temperature fell and the pain abated, but the patient was mildly delirions and extremely feeble. There was a localised pericardial

B Bulletin de la Société Anatom que, Apri], 1896

7 Bulletin de la Société Médicale des Hópitaux, Jan. 24th, 1896. 
friction rub. Ten days later be died collapsed and the necropsy disclosed a large purulent pericardial effusion, and a sero-purulent collection in the affected mertarsophalangeal joint. The pus both from the pericardium and the joint contained the pneumococcus in pure culture. The third case, described as occurring independently of pneumonia, is also related by Widal in association with Lesne. ${ }^{3}$ A man, aged 68 years, with good previous health except for slight chronic rbeumatism of his smaller joints, was seized quite suddenly with a violent chill, neadache, fever, and general aching. The next day he had pains in the whole of his left upper extremity. On the following day, the third of his illness, the pain was localised in the left sterno-clavicular joint and the back of the hand, and these parts were red and swollen. He was admitted on the eighth day when his temperature was $102 \cdot 2^{\circ} \mathrm{F}$., and there was an inflammatory swelling over the left sterno-clavicular joint and on the back of the- left hand, especially over the third and fourth metacarpal bones. The swelling on the hand gradually abated, but there was still a little puffiness when he was discharged six weeks later. The sternoclavicular joint suppurated and a small quantity of pus withdrawn on two occasions by a sterilised pipette showed a pure culture of pneumococci. The swelling was gradually absorbed without incision, but there remained some enlargement of the inner end of the clavicle.

The affection is essentially one of adult and advanced life and is far commoner in males than in females (in 28 cases there were 25 males and three females). Both these peculiarities may stand in relation to the predisposing influence of previous damage of the joint, a point to be considered later with the experimental pathogeny of the disease. The age incidence in 29 cases is as follows : from one to 10 years, two cases; from 10 to 20 years, no cases; from 20 to 30 years, one case; from 30 to 40 years, five cases ; from 40 to 50 years, nine cases; from 50 to 60 years, six cases ; from 60 to 70 years, four cases; and from 70 to 80 years, two cases. The arthritis is commoner in the upper extremity than in the lower. In 14 cases the upper extremity was alone affected; in 10 cases the lower extremity alone; in the remaining seven there was a multiple lesion affecting joints of both the upper and the lower limbs. In 19 cases inflammation was confined to a single joint; in the remainder two or more joints were involved-viz., in seven instances two joints, in two cases three, in one case four, while in the two remaining cases the arthritis was multiple and affected more than four joints. With the exception of the hip the larger joints seem more prone to be affected than do the smaller. Thus, of 41 joints affected the sterno-clavicular was concerned four times, the shoulder eight times, the elbow five times, the wrist six times, the metacarpo-phalangeal once, the hip twice, the knee 12 times, the ankle once, and the metatarsophalangeal twice. In 27 of the 31 cases, one or more of the joints suppurated, in one case the occurrence of suppuration was doubtful, and in three cases there was no suppuration, the fluid effused into the joint being of a sero-fibrinous character. In two or three cases of multiple arthritis the inflammation has been suppurative in one joint and serous in another; this was noticeably the case in the patients of Rendu ${ }^{9}$ and of Duflocq and Ledamany. ${ }^{10}$ In all of the cases included in the table, with one exception-that of Oliva-the pneumococcus was demonstrated in the fluid in the joints, but cases have been noticed of suppurative arthritis following on a recent attack of pneumonia in which the pus on examination has proved to be sterile. Widal suggests that these cases may nene the less be due to the action of the same virus, but that the coccus may have died out at the time of examination; or alternatively, that in these cases with sterile effusion the arthritis may be due to the action of toxins produced by the same organism elsewhere in the body. It is certain that some toxins of microbial origin can induce a suppurative arthritis when injected directly into a joint--e.g., the sterilised culture of the bacillus pyocyaneus (Charrin), and it is conceivable that the toxins produced by the pneumococcus in the lung may have a selective affinity for the joints in certain cases, particularly for a damaged and vulnerable joint, and produce there an arthritis with a sterile purulent effusion. But if we grant so much, how much more probable does it seem that the same toxin may

9 Ibid., June, 1900, p. 679. May 6th, 1898. induce a sero-fibrinous exudation, knowing as we do that under certain circumstances and in certain subjects the exudation induced by the living organism itself is a serous (non-purulent) effusion. It is therefore not unlikely that some cases of infective pseudo-rheumatism, characterised by a serous arthritis with sterile $\in$ ffusion, may depend upon the action of pneumococcic toxin. It is well-nigh certain that all the forms of febrile multiple arthritis now commonly classed together as "acute rheumatism" are not of the same nature, and Triboulet and Coy on ${ }^{11}$ in their most recent contribution on the subject suggest that while simple uncomplicated acute rheumarism which responds at once to salicin treatment and in which no organisms of parasitic nature have been hitherto demonstrated ${ }^{12}$ is due to the action of some unknown virus, the severe cases complicated by visceral inflammations are due primarily to the same unknown virus as the simple cases characterised by fever and arthritis alone $p l u s$ a secondary infection which may be of several different kinds, the bacillus of Achalme in some cases, staphylococci in others, and the much-discussed diplococcus in the majority. We may perbaps class Oliva's case as one of this kind due to the action of pneumococcic toxin, or we may consider it as an ordinary case of acute rheumatism accidentally complicated with pneumonia. The patient was a child, aged eight years, with the symptoms of a febrile polyarthritis which was very slightly influenced by salicylate of soda. The pyrexia did not abate and on the seventh day pneumonia supervened with pneumococci in the sputa. The pneumonia and arthritis subsided together ten days later. ${ }^{13}$ Leroux incluades this case among those of pneumococcic arthritis on, as it seems, hardly sufficient evidence, and himself admits that the record is too scanty to allow of a positive judgment. It may, however, be compared with the following case in which there was not only clinical evidence of pneumonia, but an organism, which was probably the pneumococcus, was cultivated from the blood.

A. woman, aged 24 years, was admitted to the Royal United Hospital, Bath, on March 1st, 1900. She had complained on Feb. 25th, four days before admission, of pain in her chest and indigestion. On the following day she had shivering fits followed by pain in both hips. Sbe went to bed and the pain on the same day affected her ankles, knees, wrists, and shoulders. She had had rheumatic fever three times previously, the first attack 12 years before, when she kept her bed for a month, and the last five years before. On admission her temperature was $102^{\circ} \mathrm{F}$., her pulse was 110 , and her respirations were 24 . She had a flushed face and perspiring skin, with an acid smell. The tongue had a creamy white 1 ur. Nearly all of the joints in her limbs were painful, tender, and swollen, and the more superficial joints were slightly red. The heart's apex beat was in the fifth space in the nipple-line, with a slightly increased force. There was a systolic bruit at the apex and the pulmonary second sound was accentuated at the base. She was ordered 20 grains of sodium salicylate with an alkali every four hours. She remained in much the same condition for the next few days, her temperature ranging between $99^{\circ}$ and $101^{\circ}$, and although the physiological effects of the salicylate were quite pronounced there was no abatement of the severe pain in her joints. She was very wakeful at night, and on the third day of admission she had a dose of nitrate of potash and Dover's powder at bedtime, which was repeated the next night, but each time without sleep. She also had five grains of calomel. Her mental state now became odd. She was mostly taciturn, but at times, especially after tbe visits of her friends, very excited and seemingly hysterical. A week after admission she still complained of the pain in her joints and of slight headache. She would talk continuously in a loud, slow, jerky tone and repeat some statement, usually on a religious topic, over and over again. She passed her urine several times into the bed but the motions were controlled. The urine contained a trace of albumin. There was no optic neuritis.

The salicylate, which had been reduced to 15 grains three times daily when she became delirious, was now replaced by salicin, 20 grains every three bours. Three

11 Le Rhumatisme Articulaire Aigu en Bactériologie. Paris, 1900. 12 The seven cases recently related by Poynton and Paine associated with the presence of a diplococcus were all of severe type and complicated by cardiac inflammation-so severe, indeed. that all but one proved fatal. Tre LaxoET, Sept. 22nd (p. 861) and 29th, (p. 932), 1900. 13 Oliva: Rhumatisme et Pneumococeie, Gazzetta degli Ospedali 
days later the pains were decidedly less, but she still com- perforated by the needle of a sterilised syringe. Ten cubic plained of tenderness in both hands. There had been no further incontinence of urine. She was delirious, at times noisy. On this date (March 11th) was first noted a to-andfro pericardial friction rub. Her temperature was rather higher, ranging for several days from $102^{\circ}$ to $1 C 4^{\circ}$. There was more albumin in the urine. There was no perceptible enlargement of the spleen and at no time were there any rose spots on the abdomen. Blood was drawn aseptically from a vein at the bend of the elbow. The skin was washed and scrubbed with soap and spirit lotion, with 0.1 per cent. solution of percbloride of mercury, ether, and alcohol. The skin immediately over the selected vein was frozen with a spray of centimetres of blood were withdrawn from the vein and distributed over a number of agar and blood-serum tubes. From these were grown many colonies of a diplococcus described below. The blood serum at ordinary dilutions showed no clumping influence on emulsions of bacillas typhosus. The red discs numbered 3,900,000 per cubic millimetre and the leucocytes 23,000 per cubic millimetre. On March 13th there were incontinence of urine and fæces, continuous delirium, and some complaint of pain in her head. She frequently tried to get out of bed. There was no optic neuritis. Pericardial friction was still well marked, the heart's apex heating more forcibly and as much chloride of ethyl, well seared with a red-hot rod, and as one and a quarter inches outside the nipple-line. The

Table of Cases of Preumococcic Arthritis.

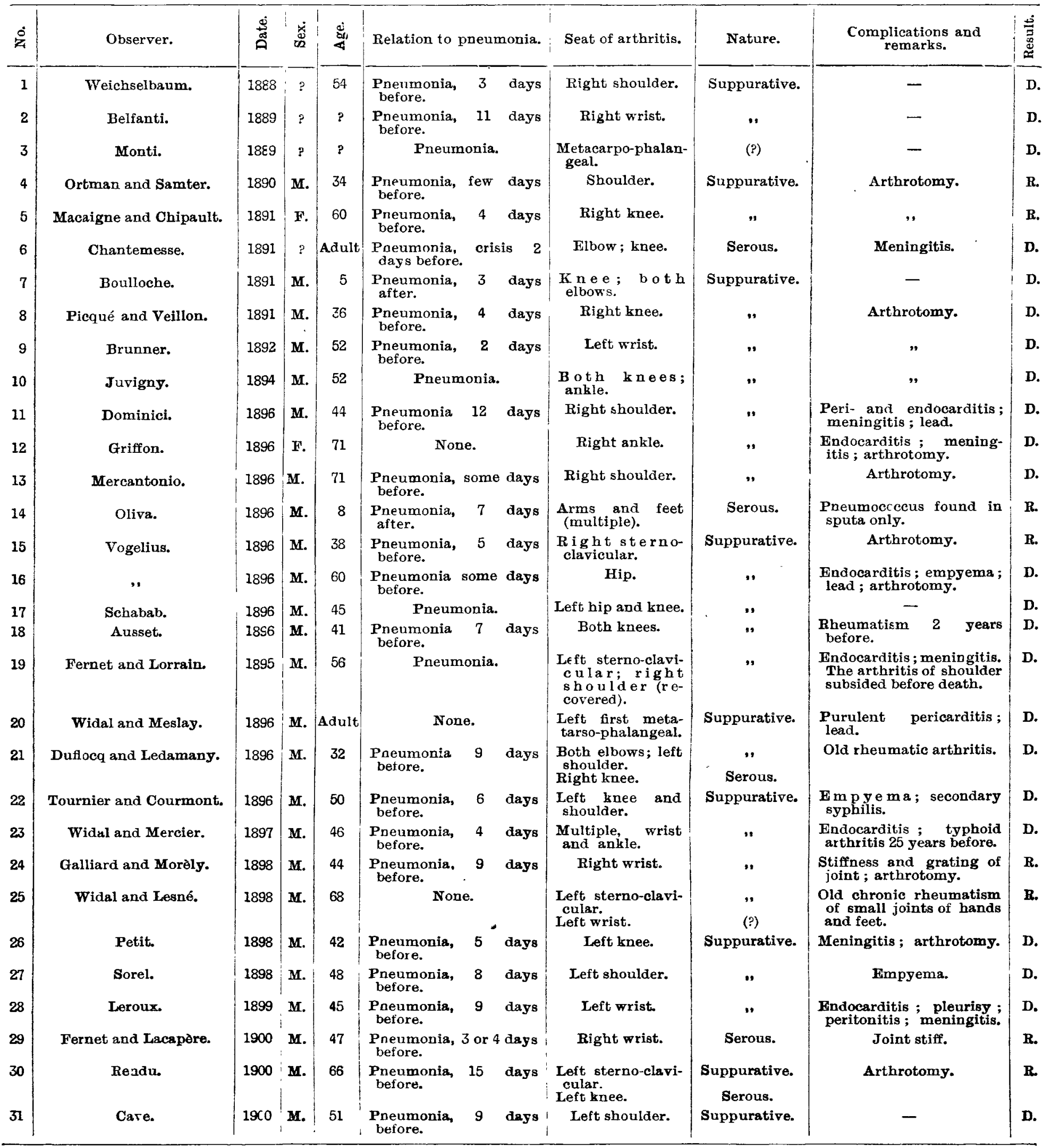


area of dulness was correspondingly increased. The following day the respirations were from 48 to 54 per minute, there were a troublesome, short, hacking cough and deep orangecoloured expectoration, viscid, and teeming with capsuled diplococci which stained by Gram. There were dulness, tubular breathing, and bronchophony at the left base to two inches above the angle of the scapula. On the 16th her condition had remained practically unchanged with incontinence of urine and fæces as before. The pulse was 136, the respirations were 44 , and the temperature was $102.5^{\circ}$. She was ordered four grains of quinia salicylas every four hours. On the 20th, four days later, her general condition showed some improvement, the temperature was lower, about $100^{\circ}$. Pericardial friction was still loud. There were dulness and crepitatio redux at the left base. On the 25th the temperature was still about $100^{\circ}$ The patient was better in herself. There was a little delirium at times and she tried often to get out of bed. The delirium was not worse at night. She complained of slight vertical headache. She was quite rational at the time of examination. She took milk well. The tongue was moist, with a thin white fur. The face was rather flushed. The pupils were equal and active. The heart's apex was in the fifth space, half an inch outside the nipple-line. The impulse was still too forcible. The area of dulness was only slightly increased upwards and outwards. There was a scratching to-and-fro friction over the whole præcordial area except at the apex, where a long systolic bruit was the only sound audible. There were still some dulness and moist sounds at the left base. There were swelling, redness, and tenderness of the knuckles of the left hand, especially of the index finger, and effusion into the right kneejoint with some tenderness. The other joints were free. On March 27th the pericardial friction was much fainter and on the 30 th it was no longer andible. The pains had disappeared from the joints. By April 5th she was much better, with a normal temperature for four or five days and no pain. There was no pericardial friction. A long swishing systolic bruit was the only sound audible at the apex and the second sonnd was accentuated at the base. The urine was now free from albumin. From this date except for a slight recurrence of pain and swelling in the joints of the right arm and hand (from April 8th to 11th) her convalescence was uninterrupted.

This case has not been included in the table as one of pneumococcic arthritis because the identification of the organism cultivated from the blood was not established by animal inoculation. Its cultured characters as tested in broth and in milk and on the surface of blood-agar and blood-serum closely resembled those of the pneumococcus, although it seemed to grow rather more freely than does the pneumococcus when recently isolated, and the colonies on agar presented a slight central opacity or haziness. No growth occurred in the cool incubator, which was kept at $21^{\circ} \mathrm{C}$. Morphologically it was a diplococcus, the individual cocci also occurring singly and in short chains. The first cultures, obtained on agar with a plentiful supply of blood, showed the presence of a distinct capsule. The cocci were round or slightly oval, measuring from 0.75 to $1 \mu$ in diameter. They were not typically lanceolate. Treated by Gram's method and stained in the anilinegentian-violet solution or carbol-crystal-violet for from 30 to 45 seconds only, they retained the stain. The organism differed from the diplococcus isolated by Poynton and Paine by this retention of Gram's stain, by its slightly greater dimensions, and by the distinct presence of a capsule. Triboulet and Ooyon, however, in describing the morphology of their diplococcus, which Poynton is inclined to regard as identical with his own, say, "Ce miorobe prend le Gram,"14 and, again, speaking of cultures in carbonated milk, " $i l$ n'est pas rare de voir parfois un espace olair donnant une apparence d'encapsulement." $1 \overline{5}$ Unfortunately the case occurred at a season of the year when professional work is heavy, and the cultures died out before an opportunity arose for more careful investigation. Still more unfortunately, working alone in a provincial town the crucial test of animal inoculation could not be applied. The case, therefore, is recorded for what it is worth as one of severe multiple serous arthritis, with pneumonia, pericarditis, and endocarditis, not influenced by salicylate of soda, and with the presence in the blood of a diplococous closely resembling (? identical with) the pneumococcus.
Turning now from the consideration of these cases of arthritis in which, while the pneumococcus has been demonstrably present and active in other parts of the body, its presence has not been detected even after skilled examination in the effusion of the affected joints, and reverting to the cases analysed in the table, we find that the mortality has been high, not so much as a direct result of the arthritis, but in relation to other distributions of the pathogenic microorganism. Of the 31 cases 23 patients died and eight recovered. In the eight recoveries are included two of the cases in which the inflammation was non-suppurative, while the remaining six were attended by suppuration. In five of these recovery ensued after incision and drainage of the joint, while in the remaining case a suppurative lesion of the sterno-clavicular joint was absorbed (the case of Widal and Lesné already referred to).

The association of the arthritis with other localisations of the pneamococcic infection is the rule, and this associated lesion is commonly in a vital part and more directly threatening to life than is the arthritis. The occurrence of pneumonia in 28 out of 31 cases has already been referred to. But of ten there has been a widespread infection of the system by the virus, with malignant endocarditis (six cases), pleurisy and empyema (five), pericarditis (two), nephritis (three), meningitis (six), and peritonitis (one). In some of the cases there has been a simultaneous inflammation of more than one serous membrane; thus in Leroux's case ${ }^{16}$ the necropsy disclosed, in addition to recent endocarditis, a purulent meningitis, pleurisy, peritonitis, and arthritis. This tendency to infect more than one serous membrane at the same time is also pronounced in certain cases of tuberculous and common septicæmic infections, and the associated multiple seritis has recently received the name of polyorromenitis. ${ }^{17}$ The morbid anatomy of the joint infected by pneumococci is essentially that of any other septic arthritis. The presence of the pneumococcus has been demonstrated in all the cases included above save one, that of Oliva, in which it was detected only in the pneumonic sputa. Its identification by chemical, morphological, and cultural characters has in most cases been confirmed by inoculation experiments, and the degree of virulence determined for mice or rabbits. The organism is found in the fluid exudation, either free or embedded in the larger cellular elements, and a thin layer of cocci may exist on the free surface of the fibrinous layer which covers the inflamed synovial membrane. The deeper parts of sections, whether of synovial membrane, cartilage, or bone, show as a rule no micro-organisms. The synovial membrane is thickened irregularly and sections stained by Weigert's method show two layers of about the same thickness; the deeper, vascular and infiltrated with embryonal cells embedded in a meshwork of connective tissue (stained pink with picro-carmine); the superficial, consisting of a network of fibrin arranged for the most part parallel to the free surface and containing leucocytes in the interstices. The lencocytes become more and more numerous towards the free surface and the fibrin less evident, except for a thin layer on the surface itself, this layer containing a number of embedded pneumococci ${ }^{18}$

In mild cases or those of short duration the synovial membrane alone may be affected, with loss of polish and injection of the fringes. But in many the cartilage is partially or completely eroded and the surface of the bone is laid bare. The changes in the cartilages begin with irritative lesions of the capsules and cells, which are swollen and cedematous-looking, while in some of the cells degenerative changes may be observed with disappearance of the nuclei, the ground substance meanwhile remaining intact. ${ }^{19}$ In the older or more virulent cases the changes are much more destructive, the ligaments and cartilages being completely destroyed, as also in some cases the articular ends of the bones. Pus may perforate the capsule and penetrate several inches along the inter-muscular planes or the sheaths of tendons. In the case of Picqué and Veillon ${ }^{2}$ it tracked upwards from the knee for six inches along the femoral artery and downwards between the muscles of the calf. One can well understand that in cases which eventually recover the joint is often left with adhesions or

17 Cf Dr. 16 Loc. cit., pp. 86-94.

18 Cr. Dr. Frederick Taylor, Brit. Med. Jour., vol. ii., 1900, p. 1693. Pneumocogne et Chipault : Remarques sur deux cas d'Arthritis Practitioner, vol, i., 1892, p. 127.

19 Widal, in Brouardel's Traité de Médecine, vol. ii..p. 785.

20 Archives de Médecine Experimentelle, January, i891, p. 68 
complete ankylosis. The milder cases with serous effasion may, however, leave behind little evidence of the preceding inflammation, and the function of the joint may be perfectly restored.

Reference has already been made to the fact that the arthritis is especially liable to be located in a joint previously damaged, whether by disease or mechanical injury. In the case herein recorded the association with a violent twist of the shoulder-joint was direct and immediate. In two other cases in the table there is the story of a fall on the affected joint shortly before the attack, in one case the shoulder and in the other the knee being affected. One patient had suffered from acute rheumatism, three from rheumatism in a chronic form, and in one of these, while the preceding rheumatism affected the knees and feet only, tive pneumococcic arthritis affected the knees. ${ }^{21}$ Two other cases were impregnated with lead, in one with the definite discovery of uratic crystals in the cartilage of the affected joint, and one patient had previously suffered from arthritis following typhoid fever. Thus, 10 cases out of 31 gave direct evidence of the situation of the infection in a previously damaged joint. This fact is of interest when we compare it with the experimental investigation of the pathogeny of the affection in animals.

To begin with, we may fairly assume, in the absence of any evidence of external inoculation of the joints, that the virus is conveyed thither by the blood, and there is ample proof of the possibility of this mode of infection. Indeed, it is notorions that in the later stages of severe pneumonia, practically in all cases that prove fatal, the pneumococous can be directly cultivated from the blood, and the writer has succeeded in doing so in several instances. In these the natural barrier to the production of septicæmia set $u p$ by the fibrinous exudation in the alveoli has been broken through with a resulting general infection. The virus circulating in the blood stream, a medium generally unfavourable to the prolonged vitality of parasitic germs, will naturally tend to settle and develop in any part of the economy where the natural defensive mechanisms of the organism are weakened by injury or disease, that is to say, in any loous minoris resistentia. Widal thus expresses himself on this head ${ }^{22}$ " Olinical experience supplies many instances of damage to the joints from internal disorders serving as a gatheringground for infection. The preceding articular lesions are sometimes clearly in evidence, such as arthritis sicca or the arthropathies of tabes or syringomyelia which become infected by various organisms. Here the predisposition to infection is favoured by the nerve lesion. The experiments of $M$. Hermann ${ }^{23}$ have shown that section of the sciatic nerve predisposed to the localisation of staphylococci circulating in the blood in different parts of the limb which was deprived of its innervation (joints, bone marrow, bursæ, and cicatrices). Recently Kasparek has repeated these researches with cultures of staphylococci, streptococci, and pnenmococci * and has shown that in animals killed after intravenons injection of the culture, pus containing the injected microbe was already present in the ankle-joint and bone marrow of the tibia on the side of the nerve lesion at the end of 24 hours. In the limb whose nerve was intact, on the other hand, there was complete absence of suppuration or, at the most, the joint contained only a few micro-organisms. Most frequently, however, the explanation of this gatheringground is not to be discovered, though in some cases careful investigation reveals an unexpected cause. Thus I have recently observed at the Hôpital Bezujon a mon-arthritis of the left first metatarso-phalangeal joint in a confirmed victim of lead impregnation who was attacked by a primary pneumococcal purulent pericarditis. At the autopsy two or three drops of sero-purulent fluid con taining pneumococci in pure culture, could be collected from the joint. By the naked eye no gouty lesion could be detected, but microscopic examination revealed the presence of crystals of urate of soda in the articular cartilages. This solitary localisation in a small joint not nsually liable to infection was only apparently fortuitous. The previous uratic deposit led to the succeeding infection. In a word, in this patient attacked by pneumococcic infection the determination to this particular joint was of a "professional' nature and should be sought in the saturnism which produced the uratic deposit." The same point,

21 Ausset : Bulletin Médical du Nord, April 24th, 1896. 22 Loc. cit.

23 Annales de l'Institu Pagteur, April 1891.

24 Wiener Medicinische Wocherschrift, August 8th and 15th, 1895. together with other details of interest, is brought out by various experiments with the pneumoccus alone. The observations of numerous investigators may be summarised as follows:-1. The injection of a virulent culture of the pneumococcus directly into a joint of a susceptible animal is almost invariably followed by acute supparative arthritis. ${ }^{26}$ 2. The subcutaneous injection of-virulent cultures after the previous excitation of an aseptic inflammation in a joint, whether by direct injury or by the injection into the joint cavity of essence of turpentine, gives varying results, in many cases negative (Gabbi), in many positive (Ausset, Zuber). 3 The intravenous injection of virulent cultures with associated aseptic traumatism of a joint leads to arthritis much more certainly than do injections by the subcutaneous method. Finally, Bezançon and Griffon ${ }^{26}$ have succeeded in producing an experimental pneumococcic arthritis by modifying the resistance of the animal experimented on in relation to the virulence of the pneumococcus. They find that if a rabbit is first partially immunised by the injection of cultures five or six days old and consequently of diminished virulence, and after a few days a full dose of a virulent culture is injected, local lesions are produced rather than a general septicæmia, and especially are these local lesions apt to appear in the joints as pneumococcic arthritis. Ieroux, who has admirably summarised these experimental investigations ${ }^{27}$ sees in this arthritis after partial immanisation a correspondence with the clinical experience that arthritis usually supervenes after a pnenmonia has run its course or towards the end of an attack when a condition of greater or less immunity has been acquired.

We can deal very briefly with the clinical characters of pneumococcic arthritis. The sy mptoms may vary in severity from pain and slight swelling limited to a single joint to an intense inflammatory cedema of the whole neighbourhood of the articulation or of a whole limb, with severe pain, heat, and redness, with abnormal mobility from destruction of the ligaments, and with grating of the bared surfaces of the bones. The fever is generally high (from $102^{\circ}$ to $104^{\circ}$ ). The nature of the arthritis can be determined only by its association with pneumonia or other pneumococcic infection and by bacteriological examination of the joint contents The prognosis is grave both as regards danger to life and the ultimate restoration of function to the joint-in the former respect on account of the extreme trequency of constitutional infection by the pathogenic organism and it characteristic localisations in the endocardinm, meninges, and other tissues previously specified. In the cases that recover progress is slow, extending over many weeks, and the function of the joint is as a rule permanently impaired.

Little that is special need be said with regard to the treatment of this particular form of arthritis. In suppurative cases-which, as we have seen, comprise the majoritythere can be no hesitation in practising aseptic incision of the joint with flushing, drainage, and fixation, as in any other acute suppurative arthritis. Those cases in which the effusion, drawn off by a sterilised syringe, has proved to be serous or sero-fibrinous in character have generally been treated by fixation on a splint with moderate compression by a bandage over cotton-wool. But even in cases of serous effu.ion it may be questioned whether an arthrotomy for the removal of the morbid products, avoiding important ligaments and tendons, would not be followed by a speedier cure and more favourable result as regards the ultimate restoration of function to the joint; while at the same time one possible source of generalised infection would be largely remored. In any case convalescence is apt to be tedious and prolonged with much stiffness and adhesion in and about the articulation. This condition must be treated on general principles, by massage, by donches and other hydrotherapeutic measures, or by hot-air baths, and when it seems necessary by forced movements of the joint; in all cases followed by suitable exercises for the restoration of wasted muscles and the preservation of the mobility that has been gained.

Bath.

25 Gabbi : Lu Sperimentale, 1889 ; Cornil, \&c. 26 Société de Biologie, July 22nd, 1899 27 Loc. cit., pp. 98-105.

Health of Cardiff. - The death-rate of Cardiff during 1900 was at the rate of 13.9 per 1000 , which is the lowest of the recorded rates for the 33 great towns. 